\title{
Polytraumatic injury with splenic rupture and unilateral avascular renal necrosis: A case report
}

\author{
Smit Shah ${ }^{1}$, Dr. Praful Shah ${ }^{2}$
}

${ }^{1}$ MS-3, Rutgers Robert Wood Johnson Medical School, NJ, USA. ${ }^{2}$ M.S. General Surgeon, Fellow of Minimal Access Surgery,

\section{Abstract}

In this paper, we present a patient who underwent an emergency exploratory laparotomy after Motor Vehicle Collison (MVC) leading to splenic injury, avascular necrosis of kidney and right upper extremity Colles' fracture. Goal of the paper is to present a patient of polytrauma along with its treatment plan in terms of prioritizing the standard of care. We also discuss various radiographical findings along with gross surgical findings that were found intraoperatively.

Keywords: Splenic, Hemoperitoneum, Colles, Blunt, Abdominal.

\section{Introduction}

Motor Vehicle Collison (MVC) are one of the leading causes of death globally. Recent study by Brown et. al. ${ }^{9}$ has demonstrated that further distance from trauma care resources has been associated with increased morbidity and mortality in patients. In addition, polytrauma in adult patients is also associated with higher risk. For instance, a study by Kanezaki et. al. ${ }^{2}$ has demonstrated that "...pelvic fractures, particularly for the elderly, most of the geriatric polytrauma patients with severe pelvic fractures were at a high risk of massive transfusion".

Furthermore, “... abnormal levels of select blood serum markers could assist in the early identification of geriatric polytrauma patients at risk for a severe medical outcome". 2 Interestingly, polytrauma has also been associated with severe systemic manifestations including hepatic dysfunction and insulin resistance. ${ }^{3}$ In this paper, we present a patient who underwent an emergency exploratory laparotomy status post MVC leading to splenic injury, avascular necrosis of kidney and right upper extremity Colles' fracture. Goal of the paper is to present a patient of polytrauma along with its proper treatment plan in terms of prioritizing the standard of care. We will also discuss the radiographical findings along with gross surgical findings that were found intraoperatively.

\section{Case Details}

Patient is a 55 year old male who was brought to emergency room after having undergone a road side accident with blunt abdominal trauma (BAT) about 30 minutes prior to arrival. Patient reported of feeling dizzy along with severe generalized abdominal pain and nonbloody, non-bilious vomiting. Vitals were as follows: BP 110/60; pulse 100; RR 20; Temp 97.8 F; Pulse Ox: 94\% $\mathrm{O} 2$ saturation on room air.

\section{Examination}

On physical exam, patient had diffuse abdominal tenderness, icteric sclera, mild ascites and pallor.

\section{Investigative Reports}

Other than minor bruises on abdomen there were no other signs of external bleeding. Guarding or abdominal rigidity were not elicited on physical exam. However, patient did have severe pain with flexion and extension of right wrist with mild swelling on the anterior aspect of the lateral of forearm.

\section{Interventions and outcome}

Given the patient's presentation, internal organ damage was suspected. Consequently, following studies were ordered: Abdominal and Pelvic CT Scan, Abdominal Ultrasound and Chest X-Ray in addition to right upper extremity X-Ray.

CT Abdomen and pelvis: Demonstrated hypo-density of right kidney which was consistent with renal hypoperfusion (Figure 1).

CT Abdomen with contrast: Demonstrated hemo-peritonuem along with splenic rupture (Figures 2 and 3 ).

Right Upper Extremity X-Ray: Demonstrated a Colles' fracture with a classic dinner fork deformity (Figure 4).

\section{Next step}

Consequently, decision was made to perform an urgent exploratory laparotomy to assess the amount of splenic bleeding and also perform a splenectomy to prevent exacerbation of hemorrhagic shock. In addition, right sided total nephrectomy was performed due to hypo-perfusion of right kidney which later demonstrated avascular necrosis.

\section{Discussion}

From a statistical stand point, trauma is a leading cause of death for age ranges 5 to 24 years and $2^{\text {nd }}$ leading cause of death in ages 25 to 65 years in US. ${ }^{5}$

Correspondence: Smit Shah; 112 Montgomery Street, Apt 3G, Highland Park, NJ 08904, USA. E-mail: spshah1991@gmail.com. 
Figure 1: Lack of enhancement of left kidney and its vasculature (Red circle) after IV contrast as compared to right kidney (Green Circle) demonstrating lack of blood flow and ischemia in avascular left renal parenchyma.
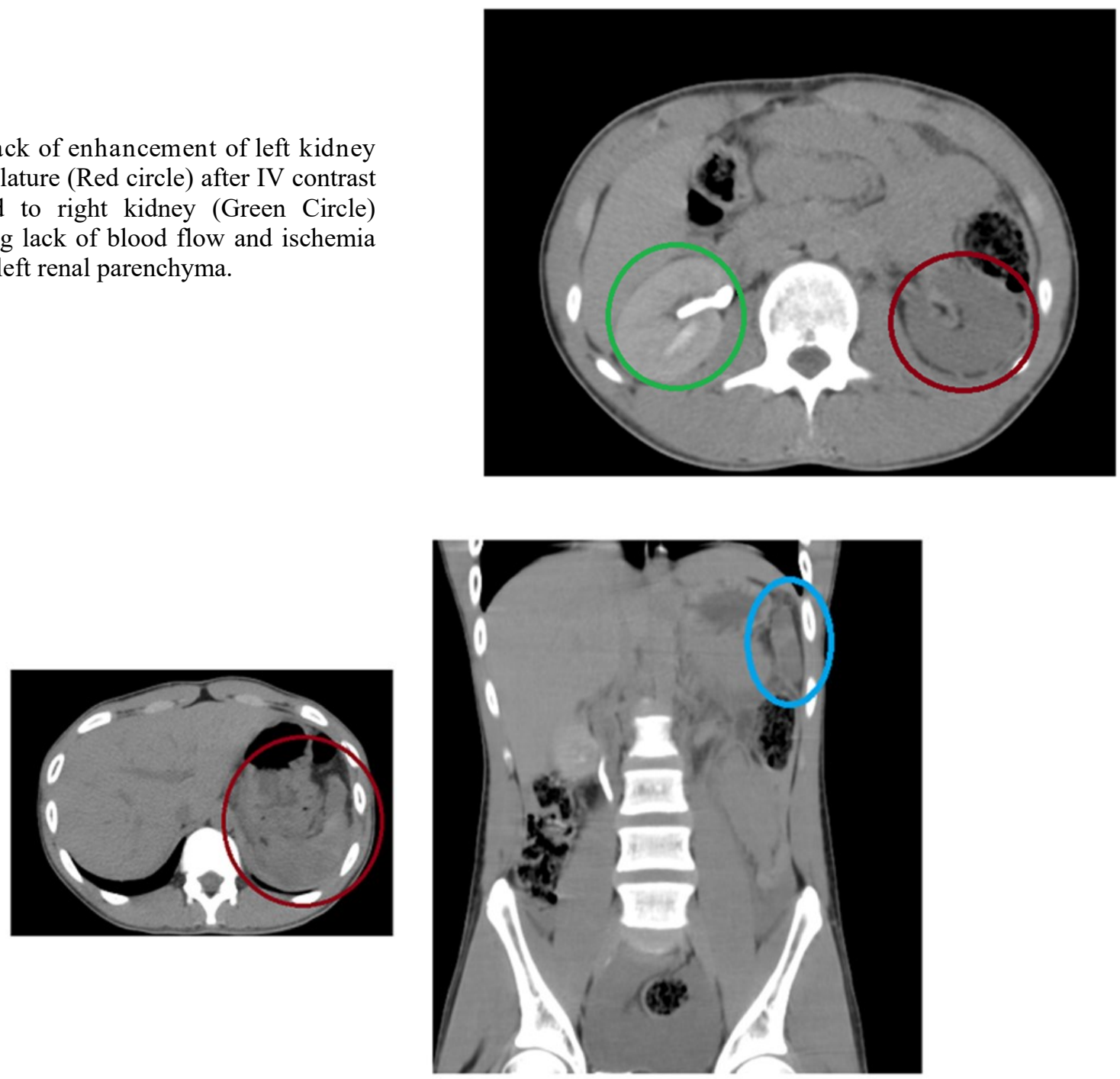

Figure 2: Red circle demonstrating ruptured spleen with peri-splenic adhesions and hemoperitoneum (Left Figure). Blue circle depicting heterogeneous appearance of spleen demonstrating varying degree of ischemia (Right Figure).

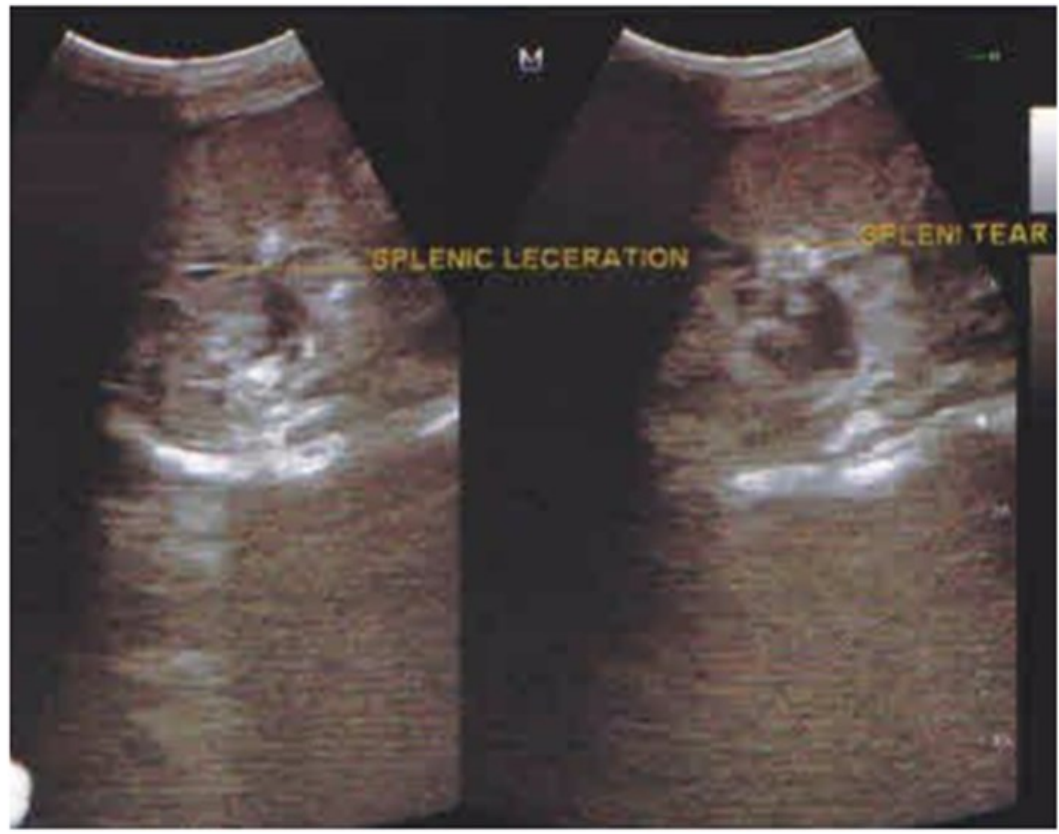

Figure 3: Splenic laceration along with tear seen on abdominal ultrasound 
Figure 4: Colles' fracture seen in the right upper extremity. We can see posterior displacement of the proximal radius and relative anterior displacement of distal radius [Classic dinner fork deformity noted].

Furthermore, "Road traffic crashes kill 1.2 million people annually around the world (3242 people a day) $90 \%$ of these deaths are in low or middle income countries. Estimated cost [is] $\$ 518$ billion globally. [Road crashes are] predicted to become the third largest contributor to the global burden of disease by 2020 ". 6 Based on the injury scoring scale from American Association of Surgery of Trauma (AAST) trauma scoring of this patient: Splenic injury was grade V since it demonstrated shattered devascularized heterogeneous spleen with hilar injury; Renal injury was grade V since it demonstrated avascularized left renal parenchyma; Chest wall injury was grade III since it demonstrated unilateral fracture of 3 adjacent ribs with flail chest (Figure 5).

From a procedural standpoint, a left subcostal incision was placed after which both spleen and left renal parenchyma were accessed. Because there was active splenic bleeding as demonstrated in Figure 1 and 2, splenectomy was performed first. After that left sided total nephrectomy was performed as demonstrated in Figure 6. Incision was closed and drain was placed which was removed 5 days after surgery. Post operatively, incision was clean, dry and intact without any exudate. On gross examination of resected left kidney (Figure 6), we can see congested specimen with swelling and edema which is in synchrony with CT findings of avascular left kidney. In addition, spleen had 3 tears on its anterolateral aspect most likely secondary

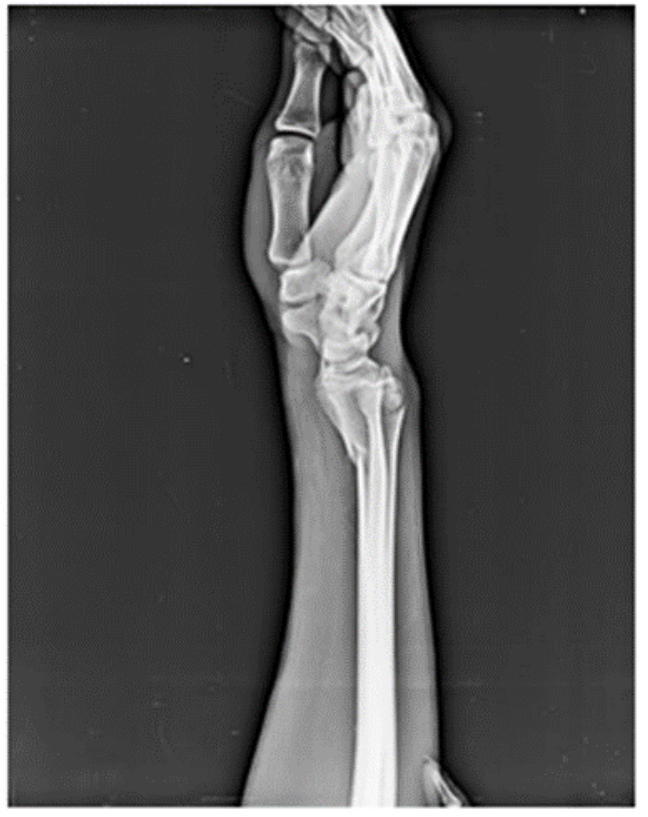

to BAT from motor vehicle crash (Figure 6). Orthopedic consult was called for left sided rib fractures and upper extremity Colles' fracture. Post operatively patient was vaccinated for Pneumococcal, meningococcal, and Haemophilus influenzae (Hib) secondary to total splenectomy in order to decrease the risk of gram negative organisms mentioned above. ${ }^{7}$ In addition, hydration with normal saline and IV cefoxitin were initiated to cover gram negative bacteria, anaerobes and enterococci was initiated. Serial blood draws of CBC (Complete Blood Count) with differential and CMP (Complete Metabolite Panel) were performed which were within normal limits over the course of hospital stay. Patient was discharged on post-operative Day 8 and was scheduled for regular follow up every 1 week which has

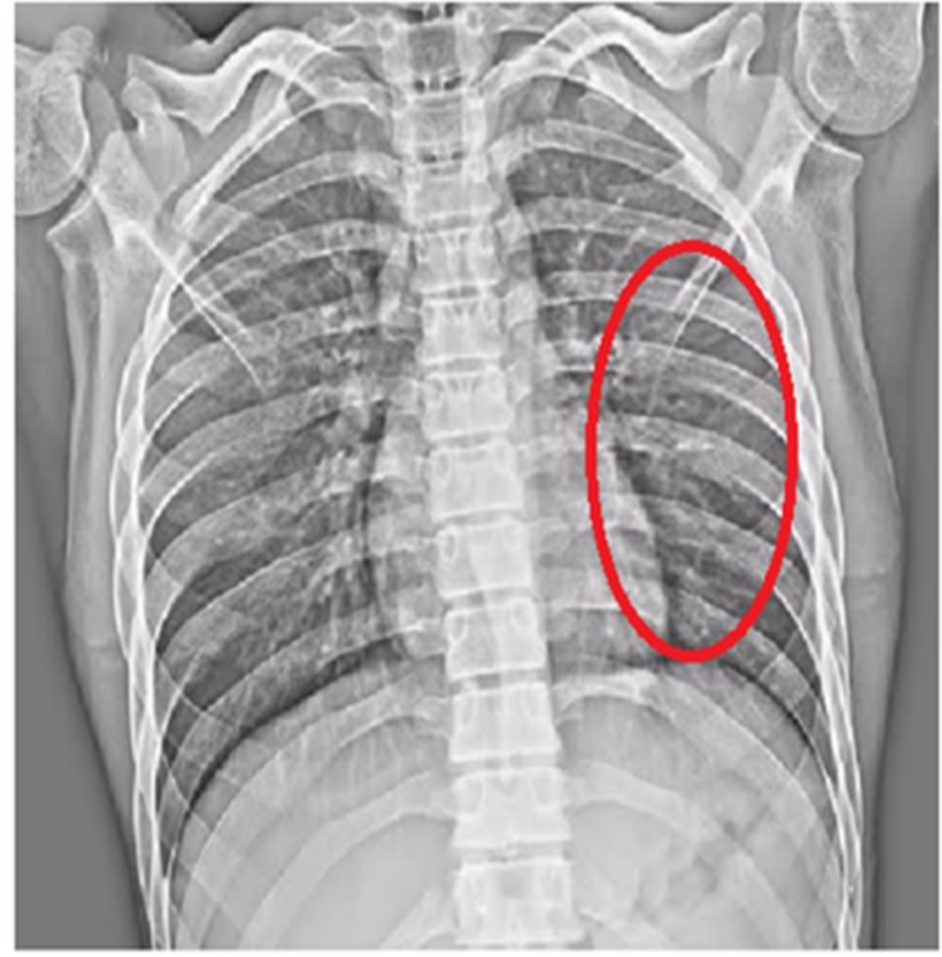

Figure 5: CXR (Chest $X$ ray) showing fractures of left $6^{\text {th }}, 7^{\text {th }}$ and $8^{\text {th }}$ ribs secondary to blunt abdominal trauma. 

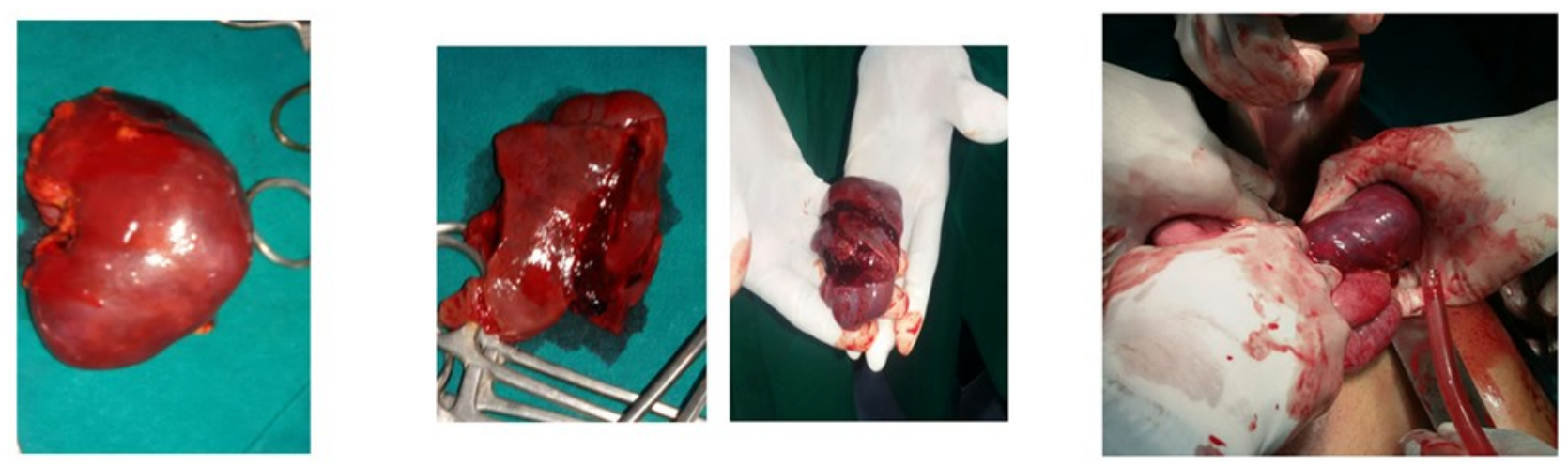

Figure 6: Intraoperative images: Demonstrating avascular resected left kidney (left); Splenic laceration (Middle Two Images) and removal of avascular left kidney (Right)

normal until now.

\section{Conclusion}

We have discussed a case of Polytrauma in an adult patient status post MVC who underwent successful total splenectomy and nephrectomy. In addition, we also discussed the radiographical findings along with gross surgical findings that were found intraoperatively.

\section{References}

1. Brown J, Rosengart M, Billiar T, Peitzman A, Sperry J. Distance matters: Effect of geographic trauma system resource organization on fatal motor vehicle collision. J Trauma Acute Care Surg 2017; 83(1):111-18.

2. Kanezaki S, Miyazaki M, Notani N, Tsumura H. Clinical presentation of geriatric polytrauma patients with severe pelvic fractures: comparison with younger adult patients. Eur Orthop Surg Traumatol 2016;26(8):885-90.

3. Akscyn R, Franklin J, Gavrikova T, Messina J. Polytrauma-induced hepatic stress response and the development of liver insulin resistance. Biochim Biophys Acta, 2017;1863(10Pt B):2672-
9.

4. The American Association for the Surgery of Trauma. Injury Scoring Scale. A Resource for Trauma Care Professionals. http://www.aast.org/ library/traumatools/

injuryscoringscales.aspx\#spleen (accessed 30 June 2017)

5. Centers for Disease Control and Prevention, National Center for Injury Prevention and Control. Leading Causes of Death Reports, 1981-2015. https:// webappa.cdc.gov/sasweb/ncipc/leadcause.html (accessed 30 June 2017)

6. Centers for Disease Control and Prevention, National Center for Injury Prevention and Control. Traumatic Brain Injury in the United States: A Report to Congress. Atlanta: CDC, 1999.

7. Webb $\mathrm{C}$, Crowell $\mathrm{K}$. Which vaccinations are indicated after splenectomy. J Fam Pract 2006;55 (8):711-2. 